\title{
Non-invasive Image-Based Approach for Early Detection of Acute Renal Rejection
}

\author{
Fahmi Khalifa ${ }^{1}$, Ayman El-Baz ${ }^{1, \star}$, \\ Georgy Gimel'farb² ${ }^{2}$, and Mohammed Abu El-Ghar ${ }^{3}$ \\ ${ }^{1}$ Bioimaging Laboratory, Bioengineering Department, \\ University of Louisville, Louisville, KY, USA \\ Tel.: (502)-852-5092 \\ aselba01@louisville.edu \\ ${ }^{2}$ Department of Computer Science, University of Auckland, Auckland, New Zealand \\ ${ }^{3}$ Urology and Nephrology Department, University of Mansoura, Mansoura, Egypt
}

\begin{abstract}
A promising approach for the automatic classification of normal and acute rejection transplants from Dynamic Contrast Enhanced Magnetic Resonance Imaging (DCE-MRI) is proposed. The proposed approach consists of three main steps. The first step segments the kidney from the surrounding abdominal tissues by a level-set based deformable model with a speed function that accounts for a learned spatially variant statistical shape prior, $1^{\text {st }}$-order visual appearance descriptors of the contour interior and exterior (associated with the object and background, respectively), and a spatially invariant $2^{\text {nd }}$-order homogeneity descriptor. In the second step, to handle local object deformations due to kidney motion caused by patient breathing, we proposed a new nonrigid approach to align the object by solving Laplace's equation between closed equispaced contours (iso-contours) of the reference and target objects. Finally, the perfusion curves that show the transportation of the contrast agent into the tissue are obtained from the segmented kidneys and used in the classification of normal and acute rejection transplants. Applications of the proposed approach yield promising results that would, in the near future, replace the use of current technologies such as nuclear imaging and ultrasonography, which are not specific enough to determine the type of kidney dysfunction.
\end{abstract}

\section{Introduction}

In the United States, approximately 12,000 renal transplants are performed annually [1, and considering the limited supply of donor organs, every effort is made to salvage the transplanted kidney [2. However, acute rejection - the immunological response of the human immune system to the foreign kidney - is the most important cause of graft failure after renal transplantation, and the differential diagnosis of acute transplant dysfunction remains a difficult clinical problem. Recently, a fairly new imaging technique, Dynamic Contrast-Enhanced Magnetic Resonance Imaging (DCE-MRI), has gained a considerable attention

\footnotetext{
* Corresponding author.
} 
for detecting acute renal rejection due to its ability to yield anatomical information. However, developing a noninvasive CAD system from DCE-MRI still a challenging problem due to the following reasons: (i) the spatial resolution of the dynamic MR images is low due to fast scanning, (ii) the images suffer from the motion induced by the breathing patient which necessitates advanced registration techniques, and (iii) the intensity of the kidney changes non-uniformly as the contrast agent perfuse into the cortex which complicates the segmentation procedures.

To the best of our knowledge, a limited work on the dynamic MRI to overcome the problems of registration and segmentation has been done. For the registration problem, Gerig et al. 3. proposed using Hough transform to register the edges in an image to the edges of a mask and Giele [4] introduced a phase difference movement detection method to correct kidney displacements. Both of these studies required building a mask manually by drawing the kidney contour on a 2D DCE-MRI image, followed by the registration of the time frames to this mask.

For the segmentation problem, Boykov et al. 5] presented the use of graph cuts using Markov models, where the energy is minimized depending on the manually exerted seed points. Giele [4] used image subtraction to obtain a mask and closed the possible gaps by using hull function.

Following these studies, a multi-step registration approach was introduced by Sun et al. 6]. Initially, the edges are aligned using an image gradient based similarity measure considering only translational motion. Once roughly aligned, a high-contrast image is subtracted from a pre-contrast image to obtain a kidney contour, which is then propagated over the other frames searching for the rigid registration parameters. For the segmentation of the cortex and medulla, a level sets approach was used.

\section{Methods}

In this paper we introduce a new and automated approach (i) to segment the kidney from its background and (ii) to correct for the motion artifacts caused by breathing and patient motion. The steps of the proposed CAD system is illustrated in Fig. 1, with details outlined below.

\subsection{Segmentation}

Geometric level-set based deformable models are popular and powerful tools in segmenting medical images because of the flexibility and independence of parameterizations of an evolving contour on the $x y$-plane [7. The object-background boundary at each moment $t$ is represented by a zero level $\phi_{t}(x, y)=0$ of an implicit level-set function - a distance map $\phi_{t}(x, y)$ of the signed minimum Euclidean distances from every point $(x, y)$ to the boundary (negative for interior and positive for exterior points). The distance map is evolved iteratively as [8]:

$$
\phi_{n+1}(x, y)=\phi_{n}(x, y)-\tau V_{n}(x, y)\left|\nabla \phi_{n}(x, y)\right|
$$




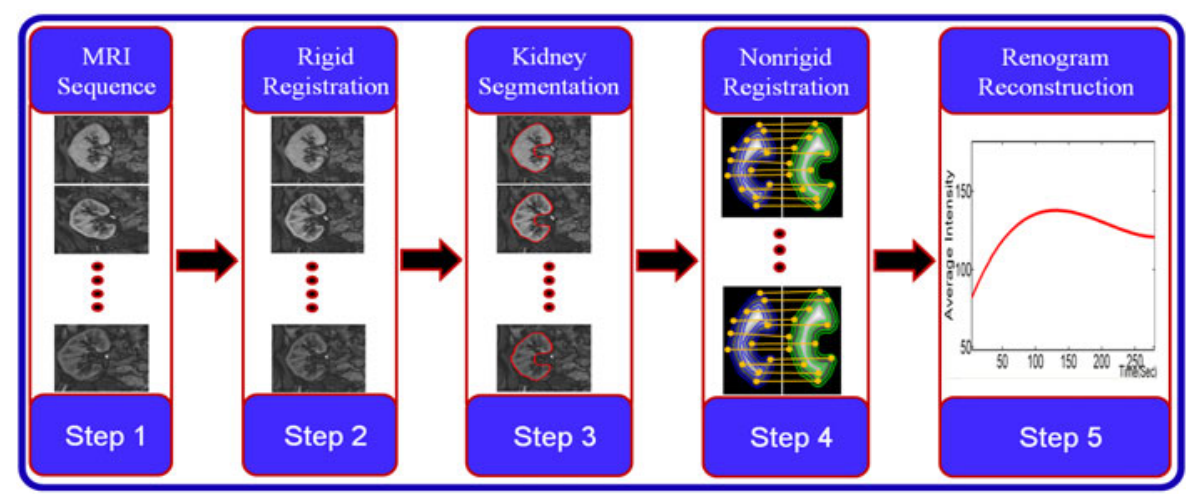

Fig. 1. The proposed CAD system

where $n$ denotes the time instants $t=n \tau$ with step $\tau>0 ; V_{n}(x, y)$ is a speed function guiding the evolution, and $\nabla \phi_{n}=\left[\frac{\partial \phi_{n}}{\partial x}, \frac{\partial \phi_{n}}{\partial y}\right]$ is the gradient of $\phi_{n}(x, y)$.

Unfortunately, conventional speed functions accounting for image intensities (e.g., 9]), object edges (e.g., [10]), an intensity gradient vector flow (e.g., [1]), etc., are unsuccessful on very noisy images with low object-background intensity gradients. Additional prior shape knowledge (e.g., [12]) results in more effective segmentation. But, speed functions based solely on the shape priors lead to large segmentation errors under discontinuous object boundaries, large image noise, and image inhomogeneities.

To overcome these limitations, a new speed function (introduced in our recent work [13]) combining the mean curvature of an evolving contour with a spatially variant joint Markov- Gibbs Random Field (MGRF) model of a gray scale image, $\mathbf{g}$, and its binary object-background map, $\mathbf{m}$, is used to control the evolution magnitude and direction at every step. The speed function in Eq.(1) is $: V(x, y)=\vartheta(x, y) \kappa$; where $\kappa$ is the mean contour curvature and $\vartheta(x, y)$ specifies the evolution magnitude and direction [13]:

$$
\vartheta(x, y)=\left\{\begin{aligned}
&-P_{1: x, y} \text { if } P_{1: x, y}>P_{0: x, y} \\
& P_{0: x, y} \text { otherwise }
\end{aligned}\right.
$$

where

$$
\begin{aligned}
& P_{1: x, y}=\frac{W_{1: x, y}}{W_{1: x, y}+W_{0: x, y}} ; P_{0: x, y}=1-P_{1: x, y} \\
& W_{1: x, y}=p(q \mid 1) p_{\mathrm{h}: x, y}(1) p_{\mathrm{s}: x, y}(1) \\
& W_{0: x, y}=p(q \mid 0)\left(1-p_{\mathrm{h}: x, y}(1)\right)\left(1-p_{\mathrm{s}: x, y}(1)\right)
\end{aligned}
$$

and $p(q \mid 1)$ and $p(q \mid 0)$ are the object and background marginal densities obtained by the LCDG model [14], respectively and $q$ is the gray level value at location $(x, y) ; p_{\mathrm{h}: x, y}(1)$ is the probability of transition to the object label in the Markov Random Filed (MRF) Potts model at $(x, y)$ at the current evolution step; and 
$p_{\mathrm{S}: x, y}(1)$ and $p_{\mathrm{S}: x, y}(0)=1-p_{\mathrm{S}: x, y}(1)$ are the empirical object-background probabilities for the manually segmented training images co-aligned by a rigid $2 \mathrm{D}$ transformation maximizing their mutual information [15, respectively. (please see 13 for more comprehensive details).

A step by step illustration of estimated densities using the LCDG model [14 is illustrated in Fig. 2 and some segmentation results for different MRI kidney images and their associated errors are shown in Fig. 3. Table 2 presents segmentation results for the MRI kidney images for ten of the subjects employed in this study (sample of the testing data).
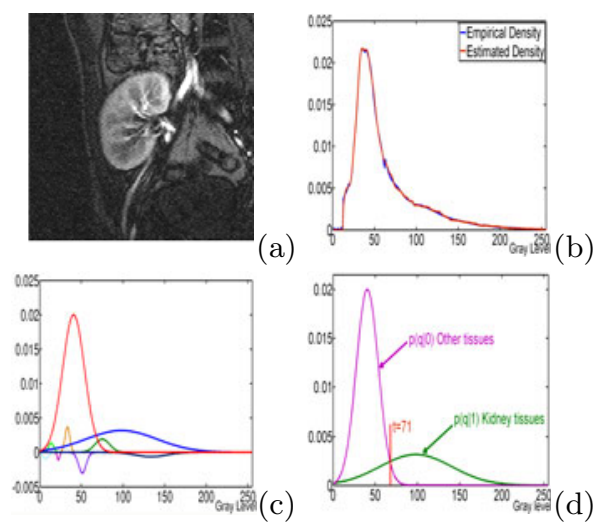

Fig. 2. (a) Typical MR kidney image, its gray level density estimation (the final estimated density (in red) for the empirical density (in blue)), (c) LCDG components, and (d) the final marginal estimated densities using the LCDG model $14(\mathrm{~d})$.
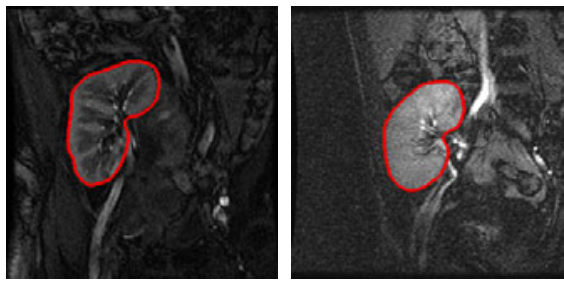

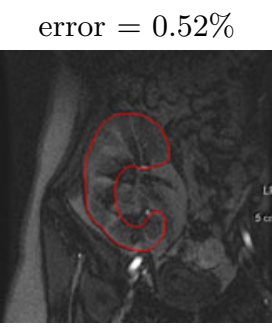

error $=1.27 \%$

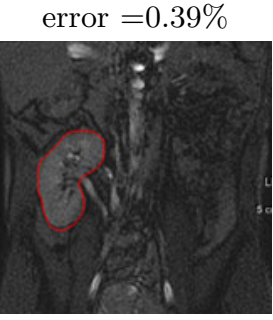

error $=1.83 \%$
Fig. 3. Segmentation results using the proposed approach. Errors are w.r.t the radiologist segmentation.

\subsection{Nonrigid Registration}

The registration is the main issue in DCE-MRI sequences. This is due to patient \& breathing movements. Although a tremendous number of nonrigid image registration techniques (e.g., 16 17,18]) have been developed, more robust, efficient, and sophisticated registration techniques are required. The centerpiece of this paper is the application of Laplace's equation in a nonrigid registration approach to find the corresponding pixel pairs between target and reference objects. Mathematically, Laplace's equation is a second-order partial differential equation for a scalar field $\Psi$ that is enclosed between two surfaces (e.g., $A$ and $B$ in Fig. 4) and takes the form:

$$
\nabla^{2} \Psi=\frac{\partial^{2} \Psi}{\partial x^{2}}+\frac{\partial^{2} \Psi}{\partial y^{2}}=0
$$




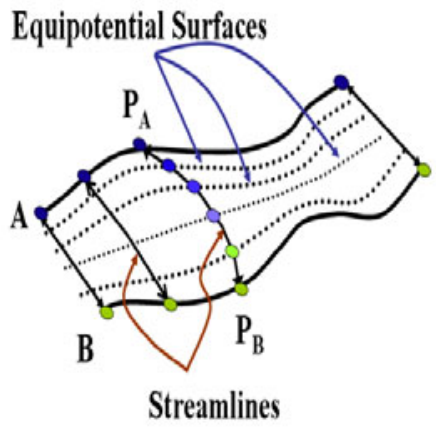

Fig. 4. Two dimensional example of the Laplace method
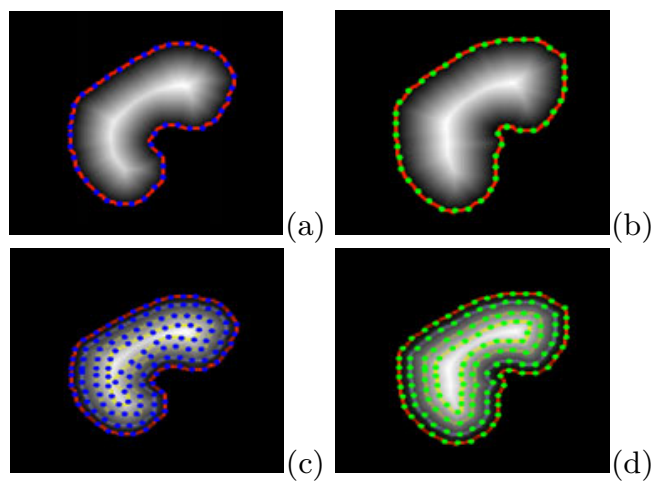

Fig. 5. The distance maps $(\mathrm{a}, \mathrm{b})$ and the isocontours $(\mathrm{c}, \mathrm{d})$ of the two kidneys

Solving Laplace's equation between the enclosed surfaces results in intermediate equipotential surfaces (dashed lines in Fig. 4) and field lines (streamlines) that connect both surfaces $A$ and $B$. These field lines are defined as being everywhere orthogonal to all equipotential surfaces (e.g., the line connecting the points $P_{A}$ and $P_{B}$ in Fig. 4). Nonrigid registration based on Laplace's equation effectively overcomes patient movement \& breathing problems and consists of the following steps:

1. First, generate the distance maps inside the kidney regions as shown in Fig. 5 (a, b).

2. Second, use these distance maps to generate equispaced separated isocontours as shown in Fig. 5 (c, d).

3. Third, solve Eq. (3) between respective iso-contours to find the corresponding pixel pairs in both target and reference objects.

In general, the number of iso-contours depend on the accuracy and the speed which the user needs to achieve. An illustration of the power of Laplace's equation to find the corresponding pixel pairs is shown in Fig. 6. The first case employs no cross-overlap between the target and reference images' edges (Fig. 6 (a, b)), and the second case employs a cross-overlap between the images' edges (Fig. 6 (c, d)). Figure. 7 shows a two dimensional illustration of how the solution of Laplace's equation is used to determine the corresponding pixel pairs. A potential $\Psi$ is found in the interior area enclosed by both surfaces $A$ and $B$ in Fig. 7 by solving Eq. (3) such that $\Psi$ is maximum at $A$ and minimum at $B$. Then the electric filed vectors, $E_{x}=\frac{\partial \Psi}{\partial x}$, and $E_{y}=\frac{\partial \Psi}{\partial y}$, in the interior area between $A$ and $B$ are used to find the corresponding pixel pairs as shown in Fig. 7 . An enlarged portion of Fig. 7 (a) around a streamline is shown in Fig. 7 (b), and a checkerboard visualization for one kidney object before and after applying the nonrigid registration is shown in Fig. 8 (a) and (b), respectively. 


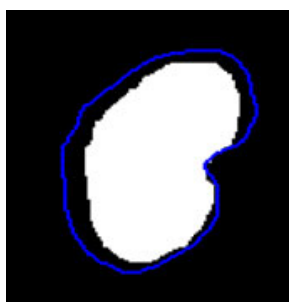

(a)

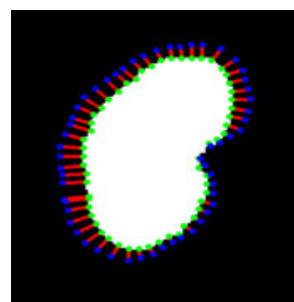

(b)

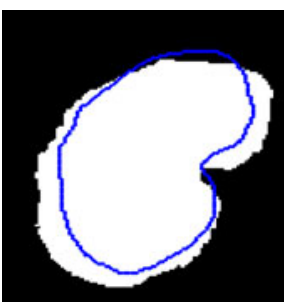

(c)

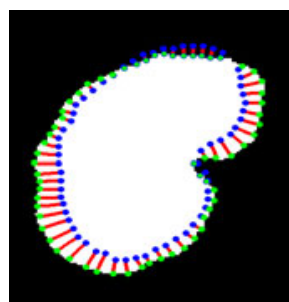

(d)

Fig. 6. Rotated kidney objects (a, c) with respect to the reference image edges (in blue), and the solution of Laplace's equation (b, d) to find the corresponding pixels.

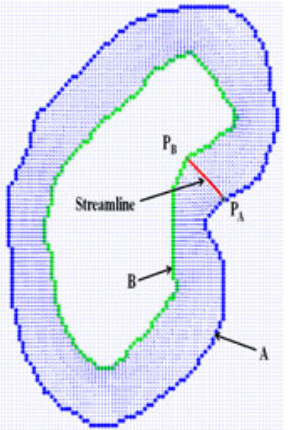

(a)

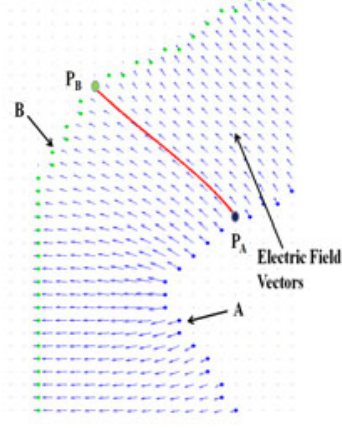

(b)

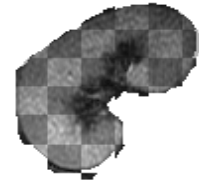

(a)

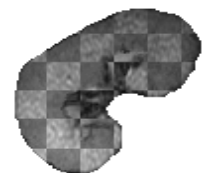

(b)
Fig. 7. Electric filed vectors in the area between two inclosed regions A (in blue) and B (in green) (a), an enlarged section around the indicated streamline (b)
Fig. 8. Checkerboard visualization before (a), and after (b) registration

Table 1. Accuracy of the proposed registration algorithm (all units are in $\mathrm{mm}$ )

\begin{tabular}{|l|c|c|c|}
\cline { 2 - 4 } \multicolumn{1}{c|}{} & \multicolumn{3}{c|}{ Simulated Displacement } \\
\cline { 2 - 4 } \multicolumn{1}{c|}{} & Small & Moderate & Large \\
\hline Maximum Simulated Displacement & 1.70 & 0.70 & 19.99 \\
Average Simulated Displacement & 0.60 & 2.30 & 9.10 \\
St. dev. of Simulated Displacement & 0.40 & 10.80 & 1.10 \\
\hline & \multicolumn{3}{|c|}{ Alignment Error } \\
\hline Maximum Alignment Error & 0.50 & 1.21 & 1.71 \\
Average Alignment Error & 0.30 & 0.90 & 1.15 \\
St. dev. of Alignment Error & 0.21 & 0.31 & 1.13 \\
\hline
\end{tabular}


Validation of the Proposed Local-Registration Approach: To validate the proposed local registration, we simulated local deformations on the real DCEMRI data set using the free form deformation (FFD) approach [16] that simulates local displacements with $2 \mathrm{D}$ cubic B-splines. To measure the registration accuracy, three different types of deformation fields, namely, with small, moderate, and large deformations indicated in Table 1 were generated with the FFD. Our registration model has been applied to each type of deformation, and the accuracy has been quantitatively assessed by comparing the simulated and recovered pixel displacements.

\section{Results and Conclusions}

The ultimate goal of the proposed algorithms is to successfully construct the renograms (mean intensity signal curves) from the DCE-MRI sequences, showing the behavior of the kidney as the contrast agent perfuses into the transplant. In acute rejection patients, the DCE-MRI images show a delayed perfusion pattern and a reduced cortical enhancement. We tested the above algorithms on twentysix patients, four of whom are shown in Fig. 9. The normal patient shows the expected abrupt increase to the higher signal intensities and the valley with a small slope. The acute rejection patients show a delay in reaching their peak signal intensities. From these observations, we have been able to conclude that the time-to-peak signal intensity, and the slope between the first peak and the signal measured from the last image in the sequence are the major two features in the renograms of the segmented kidney for classification.

To distinguish between normal and acute renal rejection, we used a $k_{n}$-nearest neighbor classifier to learn statistical characteristics from the renogram of the training sets of both normal and acute renal rejection. In our approach we used $50 \%$ of the data for the training and the other $50 \%$ for testing. For testing data, $k_{n}$-nearest neighbor based classification succeeded in classifying 12 out of 13

Table 2. Accuracy of our segmentation algorithm

\begin{tabular}{|l|c|c|c|c|}
\cline { 2 - 5 } \multicolumn{1}{c|}{} & \multicolumn{4}{c|}{ Error\% } \\
\hline Subject & Min & Max & Mean & St. dev. \\
\hline First & 0.62 & 1.20 & 1.03 & 0.98 \\
Second & 0.88 & 1.81 & 1.14 & 1.05 \\
Third & 0.76 & 2.12 & 1.35 & 1.20 \\
Forth & 0.90 & 2.50 & 1.82 & 1.54 \\
Fifth & 1.01 & 2.76 & 2.10 & 1.78 \\
Sixth & 1.20 & 3.30 & 2.23 & 2.01 \\
Seventh & 0.56 & 1.05 & 0.82 & 0.90 \\
Eighth & 0.30 & 0.96 & 0.78 & 0.85 \\
Ninth & 0.23 & 0.53 & 0.38 & 0.43 \\
Tenth & 1.10 & 1.65 & 1.22 & 0.65 \\
\hline
\end{tabular}

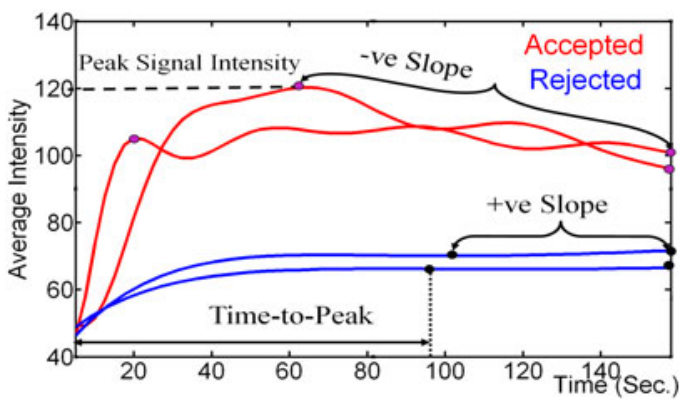

Fig. 9. Normalized average intensity from four subjects w.r.t. scan number. Subjects 1 , and 2 (in red) are normal, and Subjects 3, and 4 (in blue) are acute rejection. 
correctly $(92.31 \%)$. For the training data the $k_{n}$-nearest neighbor based classification classifies all of them correctly, so the over all accuracy of the proposed approach is $96.15 \%$.

In this paper, we presented a framework for the detection of acute renal rejection from Dynamic Contrast Enhanced Magnetic Resonance Images (DCE-MRI) that includes segmentation of kidneys from abdomen images, nonrigid registration, and $k_{n}$-Nearest Neighbor based classification. For segmentation of kidneys, we introduced a new geometric deformable model that evolves with the gray-level information of a given abdomen image, spatial information, and the shape information. Following segmentation, we introduced a nonrigid registration approach that deforms the kidney object on iso-contours instead of a square lattice, which provides more degrees of freedom to obtain accurate deformation. After nonrigid registration, the renogram of the segmented kidney for the whole sequence of a patient is calculated. The features extracted from these renograms undergo $k_{n^{-}}$ nearest neighbor based classification to understand if the transplanted kidney is undergoing acute rejection or if it is functioning normally. Applications of the proposed approach yield promising results that would, in the near future, replace the use of current technologies to determine the type of kidney dysfunction.

\section{References}

1. U.S. Department of Health and Human Services. Annual report of the U.S. scientific registry of transplant recipients and the organ procurement and transplantation network: transplant data: 1990-1999. Bureau of Health Resources Department, Richmond, VA (2000)

2. Neimatallah, M., et al.: Magnetic resonance imaging in renal transplantation. J. Magn. Reson. Imaging 10(3), 357-368 (1999)

3. Gerig, G., et al.: Semiautomated ROI analysis in dynamic MRI studies: Part I: image analysis tools for automatic correction of organ displacements. IEEE Trans. Image Process. 11(2), 221-232 (1992)

4. Giele, E.: Computer methods for semi-automatic MR renogram determination. Ph.D. dissertation, Dept. Elec. Eng., Eindhoven Univ. of Techno., Eindhoven (2002)

5. Boykov, Y., et al.: Segmentation of dynamic N-D data sets via graph cuts using Markov models. In: Niessen, W.J., Viergever, M.A. (eds.) MICCAI 2001. LNCS, vol. 2208, pp. 1058-1066. Springer, Heidelberg (2001)

6. Sun, Y., et al.: Integrated registration of dynamic renal perfusion MR images. In: IEEE Int. Conf. Image Process. (ICIP 2004), Singapore, pp. 1923-1926 (October 2004)

7. Han, X., Xu, C., Prince, J.L.: A Topology preserving level set method for geometric deformable models. IEEE Trans. Pattern Anal. Mach. Intell. 25(6), 61-79 (2009)

8. Osher, S., Fedkiw, R.: Level Set Methods and Dynamic Implicit Surfaces. Applied Mathematical Sciences, vol. 153. Springer, Heidelberg (2006)

9. Carson, C., Belongie, S., Greenspan, H., Blobworld, J.M.: Image segmentation using expectation-maximization and its application to image querying. IEEE Trans. Pattern Anal. and Mach. Intell. 24(8), 1026-1038 (2002)

10. Ma, W.Y., Manjunath, B.S.: Edgeflow technique for boundary detection and image segmentation. IEEE Trans. Image Process. 9(8), 1375-1388 (2000) 
11. Xu, C., Prince, J.L.: Snakes, shapes, and gradient vector flow. IEEE Trans. Image Process. 7(3), 359-369 (1998)

12. Chen, K., et al.: Using prior shapes in geometric active contours in a variational framework. Int. J. Comput. Vision 50(3), 315-328 (2002)

13. Khalifa, F., El-Baz, A., Gimel'farb, G., Ousephand, R., Abu El-Ghar, M.: ShapeAppearance guided level-set deformable model for image segmentation. In: IEEE Int. Conf. Pattern Recog. (ICPR 2010), Istanbul, Turkey (August 2010) (Currently in Press)

14. El-Baz, A., Gimel'farb, G.: EM Based approximation of empirical distributions with linear combinations of discrete Gaussians. In: Proc. IEEE Int. Conf. Image Process. (ICIP 2007), San Antonio, Texas, USA, September 2007, vol. 4, pp. 373 376 (2007)

15. Viola, P., Wells III, W.M.: Alignment by maximization of mutual information. Int. J. Comput. Vision 24(2), 137-154 (1997)

16. Rueckert, D., et al.: Nonrigid registration using free-form deformations: Application to breast MR images. IEEE Trans. Med. Imag. 18(8), 712-721 (1999)

17. Studholme, C., Constable, R.T., Duncan, J.: Accurate alignment of functional EPI data toanatomical MRI using a physics-based distortion model. IEEE Trans. Med. Imag. 19(11), 1115-1127 (2000)

18. Avants, B., Gee, J.C.: Geodesic estimation for large deformation anatomical shape averaging and interpolation. NeuroImage 23(1), S139-S150 (2004) 